\section{Einschätzung des Suizidrisikos}

Eine sanktionswürdige Berufspflichtverletzung kann nicht allein deshalb angenommen werden, weil sich nach einem Suizid eines Patienten herausstellt, dass der Arzt im Rahmen einer psychiatrischen Behandlung die Suizidalität falsch eingeschätzt hat. Maßgebend ist vielmehr, wie sich die Suizidgefahr für den Arzt ex ante dargestellt hat.

Der Arzt ist im Rahmen der verfassungsrechtlich garantierten Therapiefreiheit berechtigt und verpflichtet, die ihm geeignet erscheinende diagnostische und therapeutische Behandlungsmethode auszuwählen. Gerade im Bereich psychiatrischer Behandlungen ist dem Arzt ein weiter diagnostischer und therapeutischer Beurteilungs- und Ermessensspielraums zuzubilligen.

Oberverwaltungsgericht für das Land NRW, Beschluss vom 20. April 2016 - 6t E 927/14.T

\section{Ärztliche Schweigepflicht nach dem Tod eines Patienten}

Der Arzt hat zu Lebzeiten seiner Patienten die ärztliche Schweigepflicht zu beachten. Das bedeutet, dass er in einem Zivilprozess unter Berufung auf seine Schweigepflicht in Bezug auf die Pflegebedürftigkeit seiner Patienten das Zeugnis verweigern darf, so lange die Patienten ihn nicht von der Verpflichtung zur Verschwiegenheit entbinden.

Die ärztliche Schweigepflicht reicht über den Tod der Patienten hinaus. Nach dem Tod der Patienten ist zu prüfen, ob sie zu Lebzeiten geäußert haben, dass der Arzt nach ihrem Tod schweigen soll oder ob er Angaben machen darf. Gibt es eine solche Äußerung nicht, ist der mutmaßliche Wille der Verstorbenen herauszufinden, also zu prüfen, ob sie die Offenlegung mutmaßlich gebilligt oder missbilligt hätten.

Im Rahmen der Erforschung des mutmaßlichen Willens ist dem Arzt eine weitgehende eigene Entscheidungsbefugnis einzuräumen. Er muss allerdings, wenn er sich zu einer Aussageverweigerung entschließt, eine gewissenhafte Prüfung vornehmen und im Einzelnen darlegen, auf welche Belange des Verstorbenen sich seine Verweigerung stützt.

Oberlandesgericht Koblenz, Beschluss vom 23. Oktober 2015 - 12 W 538/15 me von angstlösenden Benzodiazepinen. Während des Klinikaufenthalts war vor dem Hintergrund einer früher bestehenden Benzodiazepin-Abhängigkeit die langsame Reduzierung und schließlich die Absetzung zuvor verabreichter Benzodiazepine erfolgt. Die Nebenklägerin ging davon aus, ihr behandelnder Arzt werde ihr diese Medikamente nicht mehr verschreiben. In dieser Situation kam sie auf den Gedanken, sich an den Angeklagten $\mathrm{zu}$ wenden und sein Interesse an ihr auszunutzen, um ihn durch Aufnahme einer sexuellen Beziehung zur Verschreibung von Benzodiazepinen $\mathrm{zu}$ bewegen.

In der Folgezeit erreichte die Nebenklägerin, dass der Angeklagte ihr mehrfach die begehrten Medikamente verschrieb oder Blankorezepte überließ. Der Angeklagte besorgte sich in diesem Zusammenhang frühere Arztberichte und beriet die Nebenklägerin über eine Änderung der Medikation. Weitergehende Avancen des Angeklagten, der mit der Nebenklägerin eine Lebenspartnerschaft beginnen und ein gemeinsames Kind haben wollte, wies sie zurück. Im Rahmen des mehrere Monate dauernden Verhältnisses kam es mehrfach zu einvernehmlichen sexuellen Handlungen.

\section{Das Urteil}

Die Revision des Angeklagten führte zur Aufhebung des Urteils des LG-München II und zum Freispruch durch den BGH. Der BGH hat, anders als das LG, das Verhalten des Angeklagten als nicht strafbar angesehen und ihn deshalb freigesprochen.

Ein Missbrauch im Sinne des $\$ 174 \mathrm{c}$ Abs. 1 StGB liegt nach Auffassung des BGH nicht vor, wenn eine sich bereits in ärztlicher Behandlung befindliche Patientin von sich aus das vorhandene Interesse eines mit ihr privat bekannten Arztes an ihrer Person dazu nutzt, um sich im Rahmen einer lockeren freundschaftlichen Beziehung sonst nicht erhältliche Medikamente verschreiben zu lassen. An einem Missbrauch des Behandlungsverhältnisses fehlt es demnach, wenn die Patientin dem Arzt aufgrund ihrer beruflichen Stellung und Persönlichkeit auf Augenhöhe begegnet und der Entschluss, mit dem Arzt sexuell zu verkeh- ren, nicht auf wesentlich krankheitsbedingte Willensmängel zurückzuführen ist.

Deshalb lagen die Voraussetzungen von $₫ 174$ c Abs. 1 StGB für eine Strafbarkeit des Angeklagten nicht vor, wenngleich zwischen dem Angeklagten und der Nebenklägerin grundsätzlich ein tatbestandlich gefordertes Beratungsund Behandlungsverhältnis bestand. Dieses Verhältnis missbrauchte der angeklagte Psychiater nach Ansicht des erkennenden Senats jedoch nicht zur Vornahme sexueller Handlungen mit der Nebenklägerin. An einem Missbrauch in dem vom Gesetz vorausgesetzten Sinn fehlt es, wenn der Täter nicht eine aufgrund des Beratung-, Behandlungsoder Betreuungsverhältnisses bestehende Autoritäts- oder Vertrauensstellung gegenüber dem Opfer zu sexuellen Handlungen ausnutzt. Vorliegend begegnete die Nebenklägerin dem Angeklagten indessen aufgrund ihrer beruflichen Stellung als Staatsanwältin auf Augenhöhe. Der Angeklagte nahm demzufolge nicht illegitim eine Chance wahr, die das Vertrauensverhältnis mit sich brachte.

Entscheidend für die Beurteilung, ob ein Missbrauch vorliegt, kommt es nach Auffassung des BGH ferner auf die konkrete Art und Intensität des Verhältnisses an. In diesem Fall ließ sich die Nebenklägerin vom Angeklagten nicht medizinisch behandeln, sondern holte dessen ärztlichen Rat auf freundschaftlicher Basis unentgeltlich ein. Da sich die Nebenklägerin bereits vor Beginn des Behandlungsverhältnisses entschlossen hatte, den Angeklagten zu instrumentalisieren, stellte sich ihr Vorgehen als Ausdruck ihrer sexuellen Selbstbestimmung dar und nicht als deren Missbrauch durch den Angeklagten.

\section{Literatur beim Verfasser}

\author{
Arno Zurstraßen M.A \\ Rechtsanwalt und Mediator \\ Fachanwalt für Medizinrecht und \\ Fachanwalt für Sozialrecht \\ Aachener Straße 197-199, 50931 Köln \\ contact@arztundrecht.de \\ www.arztundrecht.de
}

